\section{Percutaneous translymphatic thoracic duct embolization for treatment of chylothorax}

\begin{abstract}
Summary. A modified technique for transabdominal, translymphatic occlusion of the thoracic duct is described. During unilateral lymphangiography an abdominal lymph vessel was punctured with a fine needle under fluoroscopic guidance, and a 4 French access to the lymph system established. The thoracic duct was successfully embolized with coils and tissue adhesive in a patient with postoperative high output chylothorax. Chylous drainage immediately decreased after the intervention, the intercostal drain could be removed after seven days. Long term follow up over a ten months period confirmed the clinical success; the patient is still free of pleural effusions.
\end{abstract}

Key words: Chylothorax - Lymphatic system - Intervention Embolization - Treatment

Pekutan-translymphatische Embolisierung der Ductus Thoracicus als Therapie für Chylothorax. Es wird eine modifizierte Technik zum transabdominellen, translymphatischen Verschluss des Ductus thoracicus vorgestellt: Während unilateralter Lymphangiographie wird ein abdominelles Lymphgefäß unter Durchleuchtungskontrolle punktiert und über einen Führungsdraht koaxial eine F4-Schleuse platziert. Bei einem Patienten mit therapieresistentem postoperativen Chylothorax wurde über diesen Zugang der Ductus thoracicus distal der Verletzungsstelle mit Spiralen und Gewebekleber verschlossen. Die über eine Thoraxdrainage drainierte Chylusmenge fiel sofort nach der Intervention ab und versiegte im Verlauf, so dass die Thoraxdrainage nach sieben Tagen gezogen werden konnte. Der Patient ist mittlerweile zehn Monate frei von Pleuralerguss bzw. Chylothorax, so dass die komplikationslose Intervention auch langfristig erfolgreich war.

Schlüsselwörter: Chylothorax - Lymphsystem - Intervention Embolisation - Behandlung

\section{Introduction}

Unrecognized injury to the main thoracic duct or its tributaries during surgery is the usual cause of traumatic chylothorax. In cases unresponsive to conservative management, surgical revision with ligation of the thoracic duct is performed. Based

Fortschr Röntgenstr 2001; 173: 580-582

(c) Georg Thieme Verlag Stuttgart · New York

ISSN $1438-9029$

\author{
H. Schild ${ }^{1}$, A. Hirner ${ }^{2}$ \\ ${ }^{1}$ Radiologische Klinik \\ 2 Klinik für Allgemein-, Viszeral-, Thorax- und Gefäßchirurgie \\ Rheinische Friedrich-Wilhelms-Universität Bonn
}

upon experimental studies, an interventional technique to occlude the thoracic duct has been described [4,5]. In the following we want to report a modified technique for percutaneous, transabdominal occlusion of the thoracic duct.

\section{Patient and Technique}

A 68-year-old patient had undergone esophageal resection for a distal carcinoma. A small amount of fluid was drained by the right chest tube postoperatively. When oral food intake was started on the 9th postoperative day, the volume of drained fluid, which turned out to be chylous, increased drastically to about $2600 \mathrm{ml}$ per day; this persisted despite placing the patient on total parenteral nutrition. Over the next days the patient's general condition deteriorated secondary to continued high output chylous leakage, such that he was considered a poor candidate for surgical revision. Eventually, after 24 days of unsuccessful conservative management, we decided to attempt interventional occlusion of the chylous leak.

A lymphogram was performed via cut down on the dorsum of the left foot; a prior attempt to catheterize a lymph vessel on the right foot had failed. After intubation of a lymph vessel (Lymphangiography Set, Cook, Mönchengladbach), about $10 \mathrm{~mL}$ of Lipiodol Ultra Fluid (Guerbet Sulzbach) were carefully injected over 45 minutes with the patient on the intervention table.

Passage of the contrast medium was intermittently observed with fluoroscopy. About an hour after start of the injection, segments of larger abdominal lymphatic ducts were opacified. Under local anesthesia a larger lymph vessel at L 1 level was transabdominally punctured with a $40 \mathrm{~cm}$ long fine needle (0.7 mm diameter; Cook, Mönchengladbach) which was armed with a F 4 vessel dilator (Fig. 1). To minimize trauma, the dilator was kept on the needle segment outside of the patient until after several needle passes - a lymph vessel was entered with the needle tip as judged by fluoroscopy. A 0.012 guide wire (Tracker Therapeutics/Boston Scientific, Ratingen) was carefully advanced until the non-floppy part was securely positioned in the lymph vessel. At this time, the distal guide wire had entered the right pleural space (Fig. 2). The dilator was advanced over the needle and guide wire into the lymphatic duct, the needle removed and a Tracker catheter system exchanged. The dilator served as an access sheath and to stabilize and stiffen the system to avoid buckling.

With the catheter in the thoracic duct, direct lymphography was performed via careful injection of water soluble contrast 


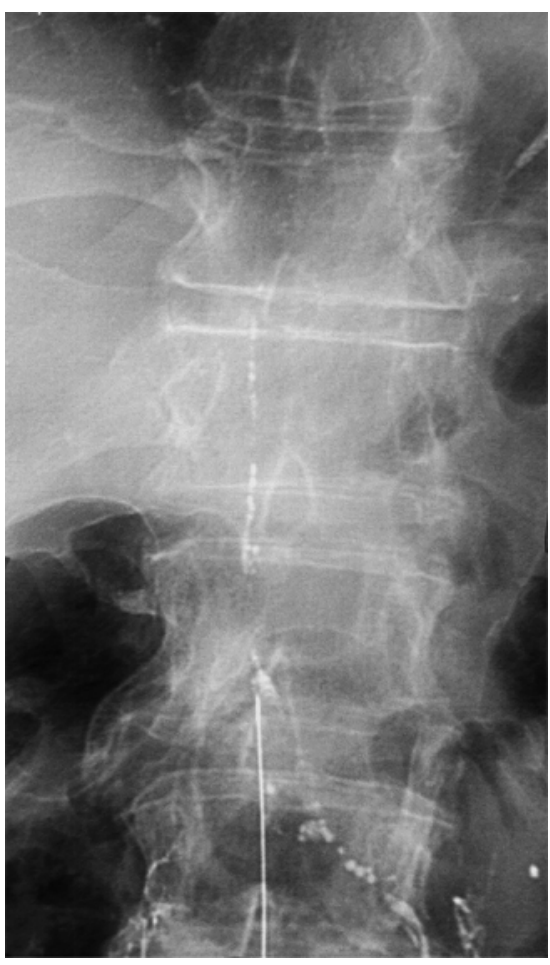

Fig. 1 Transabdominal fine needle puncture of a lymph vessel, which is segmentally opacified by lymphographic contrast medium.

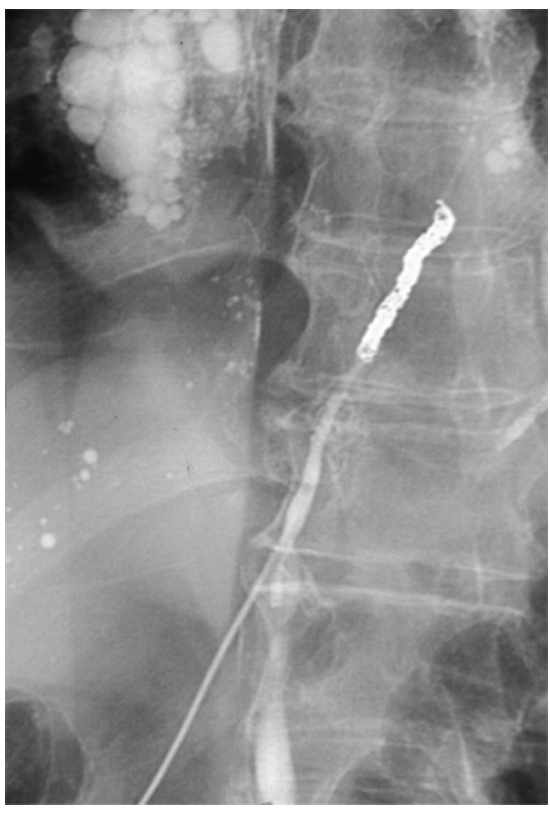

Fig. 3 The thoracic duct was embolized with seven platinum coils (3 mm diameter). Situation before injection of tissue adhesive. Note: cysterna chyli outlined by contrast medium reflux at the lower part of the image.

$0.3 \mathrm{ml}$ of a $1: 3$ mixture of bucrylate (Histoacryl, Braun Melsungen) and Lipiodol Ultra Fluid (Guerbet, Sulzbach), and immediately removed the catheter system and the dilator to avoid inadvertent fixation.

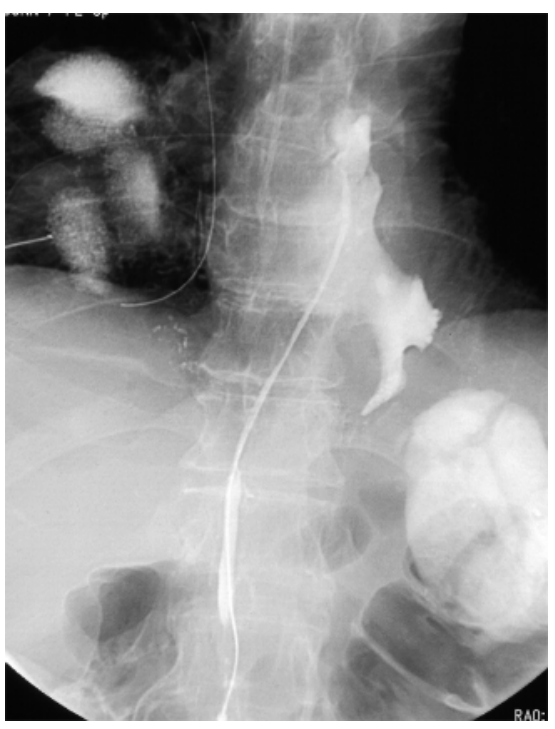

Fig. 2 The thoracic duct is outlined by contrast medium injection via the dilator, which was advanced into the punctured lymph vessel and ends at level TH 11. The guide wire enters the right pleural cavity through a defect in the thoracic duct at TH 7/8 level. After lymphography droplets of oily contrast medium are seen in both pleural spaces.

medium (Iopromide, Ultravist 300, Schering, Berlin) through the indwelling catheter. This revealed the leakage site into the right pleural space at the TH 8 level. However, some of the oily contrast medium from the transpedal lymphography was not only seen in the right, but as well in the left pleural cavity, indicating bilateral lymphatic leakage.

The catheter was pulled back to the TH 11 level well below the leakage point, where a total of seven "Tracker" coils $(3 \mathrm{~mm}$ diameter) were placed (Fig.3). Careful contrast medium injection after twenty minutes revealed only incomplete occlusion. To further embolize the duct we injected about
The patient was returned to the ward and was kept with nothing per os over night. The immediate postinterventional period (12 hours after the procedure) was uneventful, except for some mild abdominal discomfort that did not require any specific therapy, and that subsided by the next morning.

The amount of chylous drained by the chest tube decreased in the immediate post interventional period from $2300 \mathrm{~mL}$ to $170 \mathrm{~mL}$ per day. A CT study on the day after the intervention showed neither ascites nor fluid around the access site.

Oral nutrition was resumed on the second day, when $180 \mathrm{~mL} /$ 24 hrs of chylous fluid were still drained by the chest tube. Over the next days, the drainage volume continued to decrease, such that on day six after the intervention, the chest tube was removed.

After recovery from an intercurrent pneumonia, the patient was dismissed on day seventeen after intervention. Follow-up studies three and ten months after the procedure revealed neither recurrence of pleural fluids, nor accumulation of ascites.

\section{Discussion}

Injuries of the thoracic duct with chylothorax are a rare though well known complication of esophageal surgery, and are reported in 0.3 to $4 \%$ of the patients [1]. Conservative management consists of chest tube drainage, nil per mouth and total parenteral nutrition.

Surgical revision with ligation of the thoracic duct is indicated if conservative treatment fails [2]. Patients unresponsive to conservative treatment are usually those with high output 
leaks (2000 - $2500 \mathrm{ml} /$ day), and/or with no signs of reduction of the daily amount of drainage within one week of conservative treatment. Prolonged loss of major amounts of chylous fluid places the patient at a significantly increased risk of infection owing to an impaired immune competence, and is associated with a substantial deterioration of the patient's general condition. Therefore, patients with persisting loss of chylous fluids are considered poor candidates for surgical interventions. This is even more important as the surgical approach to the thoracic duct may be difficult, and has a reported mortality of up to $25 \%[1,3,6]$.

Cope et al. [4] demonstrated in animal studies that it is feasible to percutaneously catheterize the thoracic duct. Based on their experiments, they described a percutaneous approach to the abdomino-thoracic lymph system, which we modified. In our patient, a monolateral lymphangiogram via pedal cut down sufficiently opacified larger abdominal lymph ducts. Thus bilateral lyphangiography seems not to be mandatory. Because of the lymph flow, and the oily contrast medium used for lymphography, opacification of the lymph ducts was segmental and non-continuous, necessitating intermittent fluoroscopy for puncture guidance. In their report Cope et al. used an 8 French metal cannula for the puncture of the lymph duct. In our patient a 4 French dilator provided access with sufficient stability and avoided guide wire or catheter buckling.

Occlusion of the thoracic duct with multiple coils did not result in immediate and complete occlusion in our patient, which probably reflects the reduced amount of components responsible for coagulation in chyle when compared to blood. This prompted us to also seal the thoracic duct with a small amount of bucrylate. As we had to withdraw the catheter system immediately after glue injection, we could not prove that glue injection indeed helped to occlude the leakage site. As the observed reduction in drained chylous volume might have also been attributable to intraabdominal lymph leakage through the puncture site, we performed a CT study the day after the intervention. This examination, however, did not reveal any intraabdominal fluid collections, in particular not around the lymphatic puncture site at the L 1 level. We assume that the glue injection helped seal the lymphatic duct at the puncture site.

The volume of chylous drained further decreased over the next days so the chest tube was removed seven days after the intervention. The pleural effusion on the left decreased as well; only a small residual amount of pleural fluid was seen here after two weeks of follow up. Ten months after the intervention the patient is in good general condition and free of pleural and abdominal effusions.

Based on our experience, the report by Cope et al. (1999) and a report published during the review of our manuscript [7] we feel that percutaneous interventional occlusion of the thoracic duct should be considered as an alternative to surgery when (traumatic) chylothorax requires treatment. The procedure can be performed through a 4 French access; this is less traumatic than Cope's technique and may improve procedure safety.

However, percutaneous thoracic duct occlusion may be technically impossible in some patients when lymph duct anatomy precludes successful puncture, or chylous extravasa- tion comes from a small thoracic duct collateral, or from a ligated but reconstituted duct. In these cases surgery may still be required; when performed thoracoscopically, as has recently been reported [8] this may also be less traumatic for the patient than conventional operative treatment.

\section{Literatur}

${ }^{1}$ Alexiou C, Watson M, Beggs D, Salama FD, Morgan WE. Chylothorax following oesophagogastrectomy for malignant disease. Eur J Cardiothorac Surg 1998; 14: 460-466

2 Browse NL, Allen DR, Wilson NM. Management of chylothorax. Br J Surg 1997; 84: 1711 - 1716

${ }^{3}$ Cerfolio RJ, Allen MS, Deschamps C, Trastek VF, Pairolero PC. Postoperative chylothorax. J Thorax Cardiovasc Surg 1996; 112: $1361-1365$

${ }^{4}$ Cope C. Diagnosis and treatment of postoperative chyle leakage via percutaneous transabdominal catheterization of the cisterna chyli: a preliminary study. J Vas Inter Radiol 1998; 9: 727 - 734

${ }^{5}$ Cope C, Salem R, Kaiser LR. Management of chylothorax by percutaneous catheterization and embolization of the thoracic duct: prospective trial. J Vasc Interv Radiol 1999; 10: 1248-1254

${ }^{6}$ Machleder HI, Paulus H. Clinical and immunological alterations observed in patients undergoing long term thoracic duct drainage. Surgery 1978; 84: 157-165

${ }^{7}$ Hoffer EK, Bloch RD, Mulligan MS, Borsa JJ, Fontaine AB. Treatment of chylothorax: percutaneous catheterization and embolization of the thoracic duct. Am J Roentgenol 2001; 176: 1040-1042

${ }^{8}$ Wurnig PN, Hollaus PH, Ohtsuka T, Flege JB, Wolf RK. Thoracoscopic direct clipping of the thoracic duct for chylopericardium and chylothorax. Ann Thorac Surg 20001; 71: $448-450$

Prof. Dr. H. Schild

Radiologische Klinik

Sigmund Freud Straße 25

53105 Bonn

Tel. + 49-228-287-5870

Fax + 49-228-287-6093

E-mail: schild@uni-bonn.de 